\title{
DEL APRENDIZAJE SIGNIFICATIVO AL APRENDIZAJE SUSTENTABLE. PARTE 1: EL MODELO TEÓRICO
}

\section{Galagovsky, Lydia R.}

Centro de Formación e Investigación en Enseñanza de las Ciencias Facultad de Ciencias Exactas y Naturales. Universidad de Buenos Aires Ciudad Universitaria. Pabellón II. 1428 Buenos Aires. Argentina lyrgala@qo.fcen.uba.ar

\begin{abstract}
Resumen. Los enfoques constructivista y ciencia-tecnología-sociedad hacen referencia permanente a la importancia de la significación de los aprendizajes. Los términos aprendizaje significativo y su extremo opuesto, el aprendizaje memorístico, han ganado un reconocido espacio en la literatura educativa. El uso práctico de estos términos devino en una simplificación del primero, que es asociado fundamentalemnte a la enseñanza de contenidos motivantes para los alumnos, y ha desvalorizado el segundo.

En este trabajo hacemos una reconsideración de estos hechos. El modelo de aprendizaje cognitivo consciente sustentable (MACCS) que proponemos, intenta rescatar y discriminar los aspectos en conflicto y, para ello, define los términos aprendizaje sustentado, aprendizaje aislado y concepto sostén.

Palabras clave. Aprendizaje significativo, aprendizaje memorístico, aprendizaje sustentable, aprendizaje aislado, modelo de aprendizaje.

Summary. Constructivism and Science-Technology-Society approaches are particularly related to the significance of learning. Meaningful learning and its antagonist, rote learning are words highly used in educational literature. Practical usage of these words has brought about a simplification of the former, which is nowadays strongly associated with the teaching of motivational issues, and has undervalued the latter. These facts are considered in this paper. We propose a Sustainable Cognitive Model of Conscientious Learning (SCMCL) aimed at overcoming those critical points, to that end defining the terms Sustained learning, isolated learning and support concept.
\end{abstract}

Keywords. Meaningful learning, rote learning, sustaining learning, isolated learning, learning model.

\section{INTRODUCCIÓN}

En los últimos veinte años la literatura especializada en temas de enseñanza y de aprendizaje ha acuñado numerosos términos nuevos tales como constructivismo (Porlán, 1998), ideas previas, ideas alternativas y cambio conceptual (Pozo, 1987, 1997, 2001; Hewson y Hewson, 1984; Perales Palacios y Cañal de León, 2000). Recientemente, se observa el mayor uso de los términos representaciones mentales (Pozo, 2001; Riviere, 1986; Fodor, 1998) y hablar ciencia (Lemke, 1997; Jorba, Gómez y Prat, 2000).

Los diferentes autores se refieren a estos términos otorgándoles significados no idénticos (Gil Pérez et al., 1999), enmarcándolos en contextos epistemológicos particulares. A manera de ejemplo, podemos citar el caso del concepto ideas previas de los alumnos y sus sinónimos, que son tratados por diferentes autores desde, al menos, dos posturas diferentes:

- Los que consideran que son un conjunto inconsistente de conocimientos frecuentemente equivocados.

- Los que consideran que son un conjunto coherente de concepciones y teorías intuitivas, funcionales a los desempeños de cada sujeto en el mundo real.

Estas posturas conducen a actitudes docentes correlacionables, tales como: pretender erradicar tales ideas erróneas, o considerarlas barreras infranqueables, o admitir que su modificación puede ser evolutiva, o que tienen su lógica propia diferente de la de la ciencia oficial (De Posada, 2000). 
Esta diversidad encontrada en literatura abona la variabilidad de significaciones idiosincráticas que cada profesor -en tanto lector activo y reinterpretador- otorga a tales términos.

La experiencia de trabajo en capacitación docente pone en evidencia toda esta gama de significaciones alternativas que los profesores han construido en sus mentes y que les son funcionales a la hora de enseñar.

El presente trabajo se propone cuestionar ciertas significaciones que se desprenden de la teoría de la asimilación del aprendizaje (Ausubel, 1968; Novak, 1984, 1999) y que, desde nuestro punto de vista, están cobrando cuerpo en el imaginario de los profesores de ciencia. Dado que estas significaciones se asientan en cómo suponen los profesores que los alumnos aprenden, nuestro cuestionamiento es epistemológico. Una revisión crítica de tales significaciones nos permite proponer una concepción propia de aprendizaje, que presentamos como un modelo de aprendizaje cognitivo consciente sustentable (MACCS). Este modelo está enmarcado en el contexto de aprendizaje constructivista, porque sostiene que el conocimiento lo construye el alumno en su mente. El MACCS ofrece, por un lado, una visión diferente para el significado de términos tales como ideas previas, conflicto cognitivo y cambio conceptual y, por otro, recomendaciones para la enseñanza; estas cuestiones se ampliarán en un próximo artículo (Parte 2: Derivaciones comunicacionales y didácticas).

\section{REFLEXIONES ACERCA DE ALGUNAS SIG- NIFICACIONES DE LA TEORÍA DE ASIMILA- CIÓN DEL APRENDIZAJJE}

En trabajos previos (Galagovsky, 1993b, 1996; Galagovsky y Ciliberti, 1994; Muñoz, 2000; Galagovsky y Muñoz, 2002; Rodríguez, 2001; Galagovsky et al., 2003) hemos utilizado la teoría de la asimilación del aprendizaje de Ausubel como base para generar instrumentos y propuestas de enseñanza. En esta oportunidad, haremos una revisión de un punto central de dicha teoría: el papel que ocupa el discurso docente en el proceso denominado aprendizaje significativo; asimismo, propondremos el nuevo concepto de aprendizaje sustentable diferenciándolo del término aprendizaje significativo, debido a la acepción ambigua que está adquiriendo este concepto en el medio educativo argentino.

\subsection{Acerca del papel del discurso docente}

En recientes publicaciones, Novak (1999) expresa que un discurso bien organizado por parte del docente y un grupo de alumnos motivados son factores suficientes para promover que éstos alcancen un aprendizaje significativo. Evidentemente, un discurso docente mal organizado o alumnos desmotivados entorpecen enormemente las posibilidades para que ocurran aprendizajes significativos en la clase. Sin embargo, cabe preguntarse: ¿quién decide si un discurso científico (oral o escrito) está per- fectamente organizado? A nuestro juicio, esta concepción es reduccionista y otorga demasiada importancia a la estructuración de la información externa que recibiría un sujeto. Y también creemos que no hace suficiente hincapié en la necesidad de interacción dialéctica entre los estudiantes entre sí y entre el docente y los estudiantes, y en cómo estas interacciones resultan beneficiosas para el aprendizaje.

Tal como expresa De Posada (2000), la teoría de Ausubel resalta la adquisición de conceptos pero minimiza la influencia del desarrollo de las operaciones cognitivas del individuo (Lawson, 1982; White, 1988). Gil (1993) coincide en expresar que el modelo de aprendizaje significativo esboza explícitamente la asimilación de conceptos impìdiendo a los estudiantes participar en la construcción de dichos conceptos. En este modelo, el profesor asume un papel central convirtiéndose en el proveedor de conocimientos ya preparados para que los alumnos los aprendan por recepción. Detrás de esta propuesta de asimilación de conocimiento ya preparado persistiría la idea de aprendizaje inductivo. Según De Posada (2000), para sus críticos, la teoría de Ausubel continúa considerando los conceptos como algo externo al alumno que deberá ser recogido durante el proceso de aprendizaje.

La idea de Novak acerca de que un discurso docente bien organizado puede conducir a un aprendizaje significativo si tiene en cuenta los puentes cognitivos requeridos por los alumnos, nos parece frágil. Ciertamente, un discurso docente difícilmente pueda incluir los puentes cognitivos necesarios para todos los alumnos, ya que la construcción del conocimiento es idiosincrática. No cabe duda que un buen conocimiento de cuáles podrían ser esos puentes determinaría qué es necesario decir para la adecuada asimilación de ciertos conceptos. Sin embargo, esta idea nos parece peligrosa, ya que un docente que creyera en esto, estaría convencido de que él puede «decir» lo que sabe y cómo lo supo y cómo él supone que los alumnos deben aprenderlo, y con esto bastaría; el alumno, simplemente, debería aprender significativamente al escucharlo.

\subsection{Acerca de la significación del concepto apren- dizaje significativo}

Es frecuente constatar cómo los docentes trasladan el concepto de aprendizaje significativo al de contenido significativo. De esta forma, el significado del adjetivo significativo se relaciona con «algo cercano a los intereses del alumno». La consecuencia inmediata es una asociación de premisas que establece: si el contenido a enseñar está relacionado con los intereses de los alumnos, éstos estarán motivados y el aprendizaje será significativo.

Coincidimos con la línea de enseñanza ciencia-tecnología-sociedad (Solbes y Vilches, 1989; Caamaño, 1995), que hace fuerte hincapié en esta necesidad de seleccionar contenidos que «sean significativos para los alumnos», para vincular temáticas científicas abstractas a situaciones de aplicación concretas y accesibles a la realidad circundante de los diferentes alumnos. Sin embargo, 
no conocemos investigaciones que demuestren que la motivación originada por un «contenido significativo» -motivante- sea condición suficiente para lograr un aprendizaje significativo ${ }^{1}$.

Así, en el discurso educativo, el adjetivo significativo tiende a ligarse implícitamente con la motivación. Pero... ¿cuáles serían los recursos de un profesor para hacer explícita esta vinculación? (Ogborn et al., 1998). Porque también podría ocurrir que un sujeto, aun interesado en un contenido, llegara a no entenderlo, no pudiera comprenderlo.

En resumen, nuestro punto de vista crítico nos exige:

a) Reconsiderar el lugar que ocupa el discurso docente (en tanto una forma adicional de presentar parte del contenido a ser enseñado) respecto de los procesos mentales requeridos para su apropiación por parte del sujeto cognoscente.

b) Reconsiderar el concepto de aprendizaje significativo, tanto desde su origen teórico en la teoría de la asimilación del aprendizaje como desde la significación práctica que ha adquirido en el mundo educativo real.

A partir de nuestra experiencia en formación docente y de trabajo en el aula, ponemos a consideración una propuesta de aprendizaje sustentable, desde un modelo de aprendizaje abarcativo, que hemos denominado modelo de aprendizaje cognitivo consciente sustentable (MACCS). Este modelo acepta que un aprendizaje significativo sea aquél en el cual el sujeto relaciona la nueva información con aquélla que éste ya posee; pero señala requerimientos adicionales para que un aprendizaje significativo sea sustentable.

A continuación, detallaremos los puntos relevantes que caracterizan nuestra propuesta.

\section{UN MODELO DE APRENDIZAJE COGNITI- VO CONSCIENTE SUSTENTABLE (MACCS)}

El MACCS tiene cuatro aspectos fundamentales (secciones 2.1 a 2.4), que serán analizados a continuación, mediante explicaciones tanto textuales como gráficas.

La figura 1 nos permite traducir las significaciones conceptuales principales del MACCS a un formato visual, utilizando representaciones concretas (Galagovsky y Adúriz Bravo, 2001) y un lenguaje gráfico (Galagovsky et al., 2003).

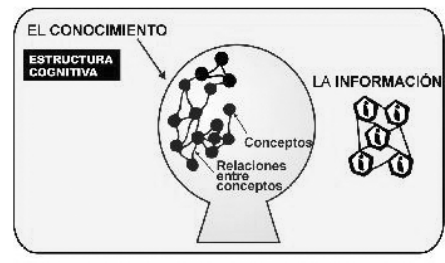

Figura 1a. Diferencias entre información y conocimiento

El conocimiento es una intrincada red de conceptos y relaciones entre conceptos que están dentro de la cabeza de un sujeto. La información está afuera de la cabeza de los sujetos.

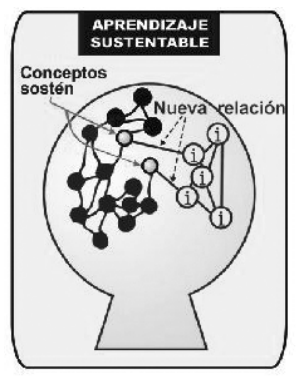

Figura 1b. Aprendizaje sustentable

Parte de la información externa es procesada y consolidada como nuevo conocimiento -círculos con la letra $i-$, vinculándose al ya existente. Los conceptos sostén -iluminados en el dibujo- son el nexo de vinculación.

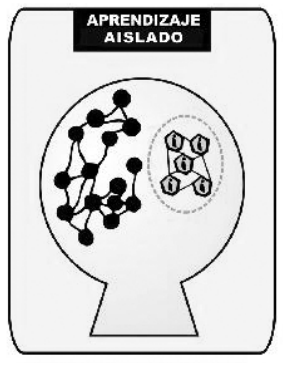

Figura 1c. Aprendizaje aislado

La información externa no es vinculada al conocimiento existente. Este tipo de aprendizaje puede evocarse y explicitarse de tal forma que un evaluador externo podría no darse cuenta de esta condición de aislamiento conceptual. 


\subsection{Diferenciación entre información y conocimiento}

Una característica distintiva del MACCS es hacer una diferenciación neta entre las significaciones de los términos información y conocimiento. La palabra conocimiento remite a lo que sabe un sujeto, a lo que tiene en su mente. La acepción vulgar del término conocimiento es mucho más amplia y remite a todo lo que una civilización, cultura o sociedad ha acuñado como saberes sobre un tema. Nuestra propuesta llama información a ese bagaje de datos, conceptos y relaciones, que están fuera de las mentes de los sujetos. Es decir, para nuestro MACCS, una sociedad o una civilización ha desarrollado conocimientos que son, en forma genérica, información; sin embargo, lo que cada sujeto guarda en su mente es su conocimiento idiosincrático.

Así, en el contexto del aula, la información es el recorte temático que el docente desea enseñar; es decir, es una selección parcial del conjunto de elementos sintácticos que existen sobre dicho tema en libros, revistas, internet, vídeos, etc., y que están expresados en diferentes tipos de lenguajes, como el verbal, gráfico, visual, matemático, etc. (Galagovsky et al., 2003). El docente recorta la información y la selecciona en función de las características del currículo oficial, de su grupo de alumnos, de la institución donde enseña, de la bibliografía y de los recursos disponibles, así como de sus preferencias.

La información en el MACCS es externa a la mente del sujeto que la tiene que aprender. El conocimiento es lo que el sujeto tiene en su mente, dentro de su cabeza. Durante el aprendizaje, parte de la información debería transformarse en conocimiento.

Nuestra concepción de información no se corresponde con la significación reduccionista que imprimen a este término algunas líneas teóricas relacionadas con la metáfora computacional y la teoría del Sistema de Procesamiento de la Información (Pozo, 2001), en las cuales ésta está codificada en lenguaje binario o puede tratarse de sílabas sin sentido. Al hablar de información estamos refiriéndonos a discursos con intencionalidad de enseñar.

Nuestra diferenciación entre información (externa al sujeto) y conocimiento (existente sólo en la mente de cada sujeto) determina un franco contraste con la teoría de la Asimilación del Aprendizaje de Ausubel. Reservamos el término conocimiento para referirnos al contenido de la estructura cognitiva de un sujeto. Si dibujáramos la estructura cognitiva como una red de conceptos y relaciones, podríamos representarla gráficamente como mostramos en la figura 1 .

Esta clara y neta distinción entre conocimiento e información es imprescindible para evitar que el docente suponga:

- que la simple exposición de dicha información logrará que ésta sea incorporada como conocimiento en las mentes de los alumnos;

- que, cuanto más información se presente a los alumnos, tanto más aumentarán sus conocimientos;

- que todos los alumnos otorgarán idénticos significados a cada una de las palabras presentes en la información que reciben; y que ese significado coincidirá con el que el docente tiene en su mente.

Llegado este punto, vemos que esta simple distinción entre los conceptos de información y conocimiento nos remite a la realidad de considerar el aspecto comunicacional de las relaciones que se producen en el aula. Los lenguajes son las interfases obligadas que separan o vinculan la información (externa al alumno) con el conocimiento que éste finalmente adquirirá. Es decir, el conocimiento que maneja un experto docente no se transmite directamente desde su cabeza a la del alumno sino que se requiere la mediación de algún lenguaje. Es así como la información nos llega necesariamente mediada por un lenguaje verbal, visual, gráfico, simbólico, gestual, matemático, etc.; y, dado que cada lenguaje tiene sus propios códigos y formatos sintácticos establecidos, es imprescindible que los docentes y los alumnos compartan esos códigos y formatos sintácticos para poder establecer una buena comunicación (Galagovsky et al., 2003).

Recíprocamente, como muestra la figura 2, el estado de conocimiento que ha adquirido el alumno (y está guardado en su mente) sólo se podrá evaluar si lo expresa y lo transforma en información.

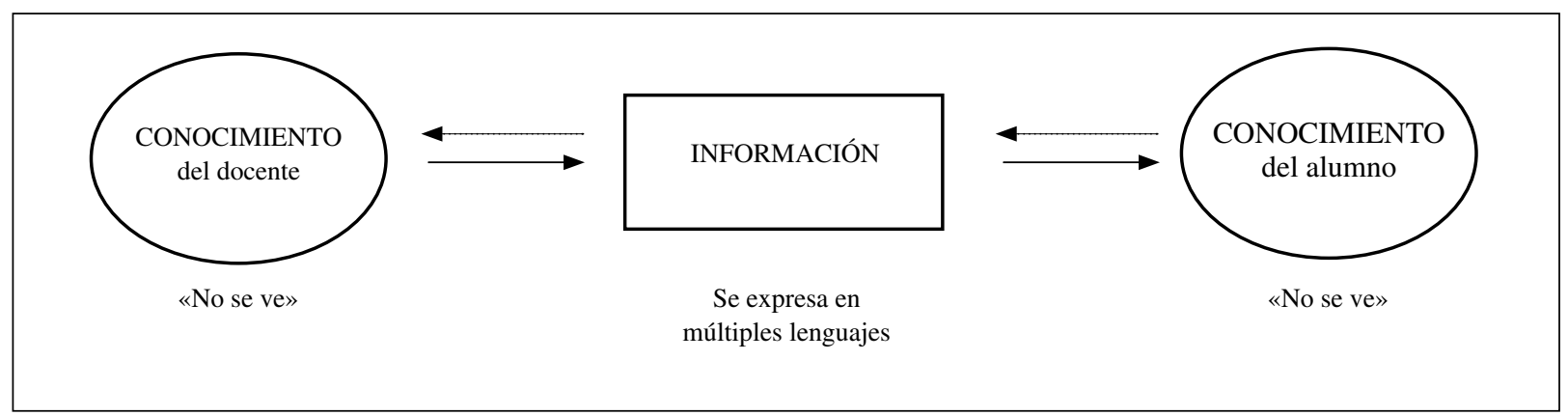


El conocimiento de un sujeto sólo existe en su cabeza y mientras no dominemos la telepatía como destreza cognitiva común, los otros sujetos no podremos «ver» ese conocimiento, a menos que se haga explícito como información.

\subsection{Diferenciación entre aprendizaje sustentable y aprendizaje aislado}

Una segunda característica del MACCS es la distinción entre dos tipos extremos de aprendizajes, el sustentable y el aislado, según se muestra en las figuras 1 b y $1 \mathrm{c}$, respectivamente.

El aprendizaje sustentable (Fig. 1b) es aquél en el que la información recibida -o parte de ella- fue apropiada como nuevo conocimiento, aumentando la red de conocimientos previos. Esta construcción nueva es, simultáneamente, una reestructuración de la estructura cognitiva previamente existente, a través de la resignificación de aquellos conceptos sostén que sirvieron de nexo para la incorporación del nuevo conocimiento.

En la figura 1b se observan ciertos códigos gráficos que se refieren a la esencia de esta definición de aprendizaje sustentable:

- Por un lado, podemos ver que la información externa a ser aprendida (Fig. 1a), toma una «forma diferente» al quedar vinculada como conocimiento en la estructura cognitiva (Fig. 1b). Esta diferencia, que implicó distorsionar los nexos y los contornos de los nodos en la representación gráfica de la información, significa que, durante el proceso de apropiación idiosincrática de la información, ocurren múltiples procesos cognitivos, entre los que podríamos mencionar: focalizar la atención, sostener la información en la memoria de corto plazo (MCP) (Mayer, 1985; Pozo, 1997, 2001), adoptar estrategias de búsqueda en la memoria de largo plazo (MLP) (Mayer, 1985), emplear estrategias de evocación desde la MLP, comparar, interpretar, resumir, clasificar, descartar, relevar, seleccionar, resignificar, vincular; integrar, diferenciar, incluir, discriminar, identificar, evaluar, valorar; elegir, emplear estrategias de guardado en la MLP. Todos estos procesos cognitivos son efectuados en la denominada memoria de trabajo (MT) (Johnstone, 1991, 1999).

- Por otro lado, la estructura cognitiva creció, se desarrolló en forma sustentable porque el nuevo conocimiento se vinculó al ya existente mediante los dos conceptos nexos, que denominamos conceptos sostén, y que aparecen «iluminados» en la figura $1 \mathrm{~b}$.

Estos conceptos sostén se resignifican al establecerse un aprendizaje sustentable, porque el sujeto que logra la sustentación de un aprendizaje ha tomado conciencia sobre la necesidad de modificar conceptos ya adquiridos, enriqueciéndolos, diferenciándolos, completándolos, reconsiderando la significación que anteriormente les había otorgado.

- Finalmente, establecer en el dibujo de la figura 1b sólo dos nexos entre el conocimiento ya existente y el que se incorporó como aprendizaje reciente es una simplificación gráfica, ya que asumimos que cuanto más vinculaciones existan entre el nuevo y el viejo conocimiento tanto más sustentable será el aprendizaje.

El aprendizaje aislado (Fig. 1c), en oposición a lo que ocurre para un aprendizaje sustentable, aparece cuando un sujeto no logra con éxito vincular una información que recibe a conocimientos previos. De esta forma, la información externa sólo podrá incorporarse a la mente del sujeto por esforzados mecanismos memorísticos de la MLP.

Este tipo de aprendizaje puede darse por varios motivos, desde la falta de motivación por desencadenar estrategias de conexión hasta no lograr ubicar, por búsqueda consciente, aquellos conceptos sostén vinculantes -porque no los tiene o porque no los encuentra en su MLP. Guardar esta información en su mente, le requerirá al sujeto esfuerzo memorístico; sin embargo, este conocimiento aislado puede también evocarse y explicitarse, incluso con un formato lingüístico idéntico al de la información de entrada (Fig. 1a), de tal forma que un observador externo podría no darse cuenta de esta condición de aislamiento conceptual.

Un aprendizaje aislado, guardado en la MLP de un sujeto, podría en algún momento relacionarse con otros conocimientos existentes en la estructura cognitiva. Es en ese momento cuando un aprendizaje aislado se tornaría aprendizaje sustentable. En tanto esto no ocurra, el MACCS prevé que este tipo de conocimiento memorístico contiene palabras o datos escasamente comprendidos por el sujeto; estas palabras no operan cognitivamente como conceptos, por lo tanto, no podrían funcionar como conceptos sostén. Esta circunstancia crítica marca un punto de inflexión que condiciona las futuras capacidades cognitivas del sujeto: los conocimientos memorísticos provenientes de un aprendizaje aislado no podrán ser utilizados como anclaje de nuevo aprendizaje sustentable. No obstante ello, el contenido de un aprendizaje aislado podría resultarle «significativo» al sujeto que lo ha memorizado si utilizáramos este adjetivo desde su significación equivalente a «tener interés en», o a «estar motivado» para querer recordarlo.

El MACCS describe dos tipos extremos de posibilidades teóricas de aprendizaje: el aprendizaje sustentable y el aislado. Los procesos de aprendizaje reales exceden al modelo teórico e implican una combinación ponderada, según cada caso, de ambos tipos extremos ${ }^{2}$.

Un aprendizaje aislado puede, como se dijo antes, convertirse en un aprendizaje sustentable mediante insight, término proveniente de la teoría de la Guestalt (Pozo, 1997), cuya traducción podría ser «visión súbita». El insight sería el proceso por el cual un sujeto toma conciencia sobre el significado de algún conocimiento que guardaba inconexo en su memoria, transformándolo en conocimiento sustentable.

Las diferencias más notables entre estos tipos extremos de aprendizaje se muestran en la tabla I. 
Tabla I

Diferencias conceptuales entre aprendizaje sustentable y aprendizaje aislado, según el MACCS.

\begin{tabular}{|c|c|}
\hline Aprendizaje sustentable & Aprendizaje aislado \\
\hline $\begin{array}{l}\text { Es un proceso que involucra capacidades y múltiples estrategias } \\
\text { cognitivas. }\end{array}$ & $\begin{array}{l}\text { Es un proceso cognitivo que sólo invlolucra habilidades } \\
\text { memorísticas. }\end{array}$ \\
\hline $\begin{array}{l}\text { Implica construcción de un conocimiento sustentable, relacionado } \\
\text { con la estructura cognitiva existente. }\end{array}$ & $\begin{array}{l}\text { No implica la construcción de un conocimiento relacionado con } \\
\text { conocimientos previamente existentes en la estructura cognitiva. } \\
\text { Origina un conocimiento aislado. }\end{array}$ \\
\hline Reconoce saberes previos en los alumnos. & No necesita recurrir a conocimientos previos \\
\hline $\begin{array}{l}\text { Necesita esfuerzos cognitivos de atención en la memoria de trabajo } \\
\text { para seleccionar y vincular conscientemente la nueva información } \\
\text { a los conceptos sostén. Requiere esfuerzo memorístico para } \\
\text { consolidarse en la MLP. }\end{array}$ & Necesita esfuerzo repetitivo para consolidarse en la MLP. \\
\hline $\begin{array}{l}\text { Puede olvidarse parcial o totalmente, pero podría ser más fácil de } \\
\text { recuperar que un aprendizaje totalmente aislado. }\end{array}$ & $\begin{array}{l}\text { Puede olvidarse parcial o totalmente. } \\
\text { Puede, algún día, vincularse y volverse sustentado, mediante insight. }\end{array}$ \\
\hline $\begin{array}{l}\text { Cuanto más se vincula, más significativo y sustentable es. La } \\
\text { información recibida es resignificada por el sujeto para transformarla } \\
\text { en conocimiento sustentable. }\end{array}$ & $\begin{array}{l}\text { La información se guarda sin vinculación a conocimientos ya } \\
\text { existentes; puede, por lo tanto, guardarse en la MLP tal cual se } \\
\text { recibió. }\end{array}$ \\
\hline $\begin{array}{l}\text { Como proceso favorece destrezas cognitivas para lograr } \\
\text { conocimientos sustentables. Éstos son anclaje y sostén para nuevos } \\
\text { conocimientos sustentables. }\end{array}$ & $\begin{array}{l}\text { Como proceso sólo favorece el entrenamiento en el uso de } \\
\text { estrategias de memoria. El conocimiento aislado no actúa de anclaje } \\
\text { o sostén para posteriores aprendizajes sustentables. }\end{array}$ \\
\hline
\end{tabular}

\subsection{Diferenciación entre los conceptos inclusores y los conceptos sostén}

Ausubel (1968) introdujo el término concepto inclusor o inclusor para subrayar el fenómeno de vinculación entre la nueva información y los conceptos ya existentes en la estructura cognitiva. Novak (1998, p. 84) dice: «Un concepto inclusor no es una especie de tira matamoscas a la que se adhiere la información, sino que desempeña una función interactiva en el proceso de aprendizaje significativo, facilitando el paso de la información relevante por las barreras perceptuales y sirviendo de base de unión de la nueva información percibida y el conocimiento previamente adquirido.»

El MACCS presenta el nuevo término concepto sostén para señalar el nexo entre la información que se está procesando como nuevo conocimiento en un aprendizaje sustentable. La diferencia teórica fundamental entre un concepto inclusor y un concepto sostén radica en que el primero puede no ser un nexo correcto; mientras que el segundo, deberá serlo.

Un ejemplo puede resultar ilustrativo. Supongamos que un sujeto asegura que «en verano hace más calor que en invierno porque la Tierra está más cerca del Sol» y realiza un esquema de la traslación de la Tierra alrededor del Sol para explicarlo. De su aprendizaje podremos decir que le es significativo porque:

- ha construido un modelo mental visual en el que ubica el eje de la Tierra perpendicular al plano de la eclíptica y ubica la Tierra en un foco de la elipse que describiría en su traslación alrededor del sol;

- argumenta con lenguaje verbal una situación que ha experimentado vivencialmente durante toda su vida y predice que esto ocurrirá siempre, ya que se basa en su amplia experiencia al respecto.

- su modelo visual y su argumento verbal le permiten predecir, año a año, un fenómeno natural que, al cumplirse, refuerza la significación y la razón de dicho modelo.

Sus conceptos inclusores serán, precisamente, los derivados de su experiencia perceptiva y su convencimiento cognitivo. Nos vemos pues, en la necesidad de decir que este aprendizaje significativo es erróneo, pues los conceptos inclusores utilizados para darle existencia son inapropiados, desde el punto de vista del conocimiento científico.

Necesitamos restringir la gama de potenciales conceptos inclusores a aquéllos que son «científicamente correctos», y en el contexto del MACCS los denominamos conceptos sostén. Esta discriminación tiene muy importantes derivaciones didácticas que se ampliarán en la continuación de este artículo (Parte 2).

\subsection{Restricción a procesos cognitivos conscientes}

El MACCS intenta hacer aportes sobre los procesos cognitivos conscientes puestos en funcionamiento con el objetivo de aprender. Hay factores de otra índole, tales 
como los afectos, los temores, los prejuicios, los intereses, las motivaciones, la realidad socioeconómica, etc. Estos factores penetran y atraviesan, permanentemente, las situaciones de aula y de aprendizaje, condicionando las habilidades cognitivas de los sujetos, al punto de ser tan importantes como para bloquear sus posibilidades de aprender; o, por el contrario, a partir de un vínculo positivo y una fluida comunicación global con el profesor, ser un estímulo vigoroso para facilitar aprendizajes sustentables. Sin embargo, dado que dichos factores son aplicables a relaciones vinculares específicas de situaciones particulares, consideramos que exceden el marco del presente modelo. Es por esto que, tanto en nuestra expresión verbal como en nuestras representaciones gráficas del MACCS (Fig. 1a, 1b, 1c), sólo hacemos referencia a la parte cognitiva consciente de la estructura de la mente. La estructura afectiva, emotiva o vincular existe, es la fuerza vital de cada sujeto y su importancia es sustancial a la hora de predisponerlo para el aprendizaje consciente, pero sus complejas variables no están representadas en el MACCS.

El MACCS describe procesos que ocurren en la zona de la conciencia. La mente humana es tan compleja que no hay teorías abarcativas que, hasta el presente, hayan logrado describirla completamente. Los abordajes psicoanalíticos establecen esferas de estudio a niveles del inconsciente y sus mecanismos; las teorías cognitivas, en cambio, no indagan en el inconsciente de los sujetos (ni Piaget, ni las teorías de procesamiento de la información, ni la teoría ausubeliana siquiera lo mencionan). Nuestro MACCS sólo describe capacidades de aprendizaje a nivel consciente; sin embargo, dejamos sentado nuestro reconocimiento de los procesos del inconsciente cuyos contenidos no son accesibles a la conciencia en forma directa.

Los procesos cognitivos conscientes que llevan a un aprendizaje sustentable requieren enfocar la atención para, por ejemplo, mantener en la MCP la información entrante, hacer búsquedas y evocaciones desde la MLP, establecer las vinculaciones y establecer el conjunto de conceptos sostén involucrados. Todos estos procesos deben ocurrir simultáneamente en la MT. El aprendizaje aislado, en cambio, requiere atención consciente para retener la información entrante en la MCP y, luego, ejercitación repetitiva hasta consolidación en la MLP.

Consideramos la estructura cognitiva (EC) de cualquier sujeto como una red tridimensional de conceptos y relaciones entre conceptos. Las representaciones gráficas del MACCS, en las figuras 1a, 1b y 1c, señalan exclusivamente aquella pequeña porción de la estructura cognitiva que, en determinado momento, se hace consciente. Para que ocurra un aprendizaje sustentable, los conceptos sostén deben estar en el nivel consciente y la vinculación entre la nueva información y dichos conceptos se hace con toma de conciencia. El resto de la red cognitiva, que no está involucrada en el momento en que ocurre dicho proceso de aprendizaje, estaría en un nivel preconscien$\mathrm{te}^{3} \mathrm{y}$, por ello, no dibujada en la figura 1.

Las limitaciones gráficas de espacio nos restringen a dibujar unos pocos nodos unidos mediante nexos para simbolizar la EC consciente involucrada en un eventual aprendizaje sustentable. Aun así, los gráficos de la figura 1 nos permiten deducir derivaciones didácticas:

- A mayor conocimiento sustentable en la EC ya existente, mayor número de conceptos sostén que serán potenciales anclajes de nuevos aprendizajes sustentables. Este punto nos permite establecer un requisito diferente de la edad o del estadio evolutivo piagetiano como indicador de la «amplitud»o «capacidad» de esa EC frente al contenido que va a aprender.

- A mayor entrenamiento en habilidades cognitivas relacionadas con la búsqueda de posibles nexos guardados en la MLP, mayor capacidad para disponer de conceptos sostén en la memoria de trabajo. Este punto nos permite entender la resistencia de muchos alumnos frente a propuestas educativas que «los hacen pensar» si sus entrenamientos en los años de escolaridad se basaron, fundamentalmente, en actitudes pasivas y pocas habilidades de este tipo.

Sabemos que existen otros tipos de aprendizajes (subliminales, inconscientes, etc.), que no están considerados en el MACCS.

\section{REFLEXIONES ACERCA DE ALGUNAS SIG- NIFICACIONES DERIVADAS DEL CONCEPTO DE APRENDIZAJE MEMORÍSTICO}

Es frecuente que los docentes expresen la necesidad de estimular un aprendizaje de tipo significativo frente a la opción polarizada entre aprendizaje significativo o aprendizaje memorístico, proveniente de la propuesta de Novak (1984, 1999). Así, el aprendizaje memorístico devino, en el discurso educativo, en algo desvalorizado, a pesar de las recomendaciones del propio Novak, que sostiene que un aprendizaje memorístico es la única opción cuando los alumnos no tienen conceptos inclusores para lograr la inclusión significativa del nuevo conocimiento.

Estas derivaciones nos llevaron a plantearnos qué papel juega la memoria en nuestro modelo:

Nuestro MACCS tiene como tercera característica el reconocimiento de que un aprendizaje sustentable también requiere esfuerzo memorístico para consolidarse en la MLP. Es decir, que una información se aprenda significativamente y en forma sustentada y pase a formar parte de nuestro conocimiento relacionado no quiere decir que, automáticamente, se retendrá en la memoria por el resto de la vida. Las estrategias de «guardado en la memoria» son demandadas para los dos tipos de aprendizaje en el MACCS. La diferencia fundamental es que la repetición memorística sería la única estrategia cognitiva requerida para lograr un aprendizaje aislado, en contraposición con la compleja red de estrategias cognitivas requeridas para el logro de un aprendizaje sustentable.

Los esfuerzos que debe hacer cada individuo para guardar una información en su MLP y luego pretender 
recordarla son absolutamente personales. La teoría de las inteligencias múltiples (Gardner, 1983) es un aporte que daría cuenta de factores idiosincráticos facilitadores u obstaculizantes de estos procesos cognitivos.

Para avanzar en la escolaridad todos hemos debido desarrollar estrategias cognitivas de guardado y recuperación de información en nuestra MLP. Actualmente, se observa sobre todo en la transición entre el segundo y el tercer ciclo de la Escuela General Básica en Argentina (11-14 años) que -posiblemente debido a la moda generalizada de menospreciar los aprendizajes «memorísticos»- muchos estudiantes, simplemente, no han desarrollado estrategias de guardado en la MLP; ni siquiera las famosas reglas nemotécnicas.

Es fundamental hacer hincapié en la importancia de no despreciar las estrategias cognitivas relacionadas con los procesos de memoria. Baste con destacar que los conceptos sostén están en la memoria; entonces, aquel sujeto que guarda muy poco conocimiento de un área del saber en su memoria, o que no está familiarizado con estrategias de búsqueda y evocación en su MLP, tendrá pocos conceptos sostén accesibles para desarrollar próximos aprendizajes sustentables.

Como se desprende hasta aquí, desde el MACCS, tanto los aprendizajes sustentables como los aislados pueden ser ambos significativos, en el sentido que los sujetos estén motivados para guardarlos en sus mentes. Ambos también requieren esfuerzos memorísticos para ser guardados en la MLP y evocados desde ésta.

\section{REFLEXIONES ACERCA DE LA EXISTEN- CIA DE ERRORES EN APRENDIZAJES SIGNI- FICATIVOS}

El discurso educativo actual ha enaltecido las palabras aprendizaje significativo, y parece implícito el hecho que un aprendizaje significativo es siempre correcto.

La postura de Ausubel y Novak no se explaya sobre este espinoso tema. Sin embargo, en todo su discurso se destila una suerte de asociación entre aprendizaje significativo y aprendizaje correcto: «Los conocimientos que se han adquirido en forma significativa, que se han aprendido a partir de la acción, el sentimiento y el pensamiento consciente, son conocimientos que se controlan. Piénsese en cualquier campo de conocimiento en que se pueda relacionar lo que se sabe con el modo en que dicho conocimiento opera para dar sentido a la experiencia en ese campo, y se tendrá un ejemplo de un conocimiento aprendido en forma significativa.» (Novak, 1999, p. 53).

Sin embargo, cabe aceptar la posibilidad que un sujeto realice un aprendizaje significativo y que éste sea erróneo cuando una nueva información se conecta con conceptos inclusores no apropiados. Desde nuestro punto de vista, consideramos que muchas investigaciones sobre ideas previas o alternativas, que las muestran como re- sistentes al cambio conceptual (Pozo, 1997), pondrían en evidencia este tipo de aprendizajes escolares significativos y erróneos. Un ejemplo típico es el que discutimos en el ítem 2.3.

Nuestra propuesta pretende que un aprendizaje sustentable sea también correcto. Durante la apropiación de la información, el sujeto debe buscar, seleccionar, confrontar, encontrar entre todos los conceptos inclusores accesibles a su mente consciente, aquellos conceptos sostén que sean apropiados ${ }^{4}$.

El docente tendrá que afinar sus propuestas de actividades para generar espacios de clase que permitan dicha toma de conciencia, la autoevaluación y el análisis metacognitivo de los alumnos. Se recomienda generar en la clase un buen clima afectivo donde se fomente la confianza en sí mismo, no se automarginen explicitaciones o argumentaciones por temor al ridículo y se ayude a tomar conciencia sobre los errores. La participación de los alumnos debe ser valorada, pero no desde el aporte exclusivo de las respuestas finales correctas expresadas por unos pocos alumnos iluminados, sino favoreciendo la explicitación de las formas idiosincráticas de los estudiantes para procesar la información que se pretende enseñar. Estos aspectos didácticos están muy entrecruzados con los aspectos vinculares que se establezcan durante la clase: si ambos son positivos predispondrán favorablemente para aprendizajes sustentables.

Las consignas precisas y el tiempo de clase necesario para dar lugar a argumentaciones comprometidas con la expresión de las representaciones mentales de los alumnos (Ximénez Aleixander, 1996) y sus formas de expresarlas (Galagovsky et al., 2003) son actividades que ayudan a la preparación consciente de los conceptos sostén apropiados. Dado que cualquier alumno, frente a las consignas del profesor, puede activar en su mente conceptos erróneos para vincular la información a ser aprendida y, por lo tanto, dar lugar a significaciones equivocadas, debe darse lugar en la clase para que los alumnos expresen lo que están procesando en sus mentes. El docente no debe evitar este tipo de situaciones, ni descartar la explicitación de conceptos inclusores erróneos, sino que debe ayudar a sus alumnos a tomar conciencia de esta situación y a encontrar -en sus mentes- los conceptos sostén apropiados. El docente que piensa en función del MACCS tratará de evitar la consolidación del error en el momento mismo en que se está formando.

Este punto diferencia al MACCS de la teoría de la asimilación del aprendizaje, ya que:

a) El MACCS involucra la toma de consciencia del sujeto que aprende sobre cuáles son los conceptos sostén apropiados para el logro del aprendizaje sustentable. Esto implica un sujeto cognoscente activo, metacognitivo, y que expresa como información circulante el contenido de la estructura cognitiva que está puesta en juego en el momento de aprender (Fig. 2). El estudiante debe explicitar sus potenciales conceptos nexo y seleccionarlos o descartarlos con argumentaciones hasta encontrar 
los conceptos sostén apropiados. Cualquier concepto nexo que el alumno traiga a su conciencia no necesariamente es el concepto vinculante correcto; si así fuera, sería muy fácil aprender.

b) El MACCS involucra a un docente atento a la información proveniente de las argumentaciones de sus alumnos, los cuales ponen en evidencia cuáles son las significaciones que están construyendo en sus mentes. Esto implica un docente que asuma el rol de provocar situaciones didácticas de interacción y discusión argumentativa entre los alumnos, hasta facilitar la aparición de los conceptos sostén pertinentes y de instancias metacognitivas permanentes para la superación de eventuales errores.

Este modelo de aprendizaje sustentable hace hincapié en la necesidad preponderante de llevar a cabo un modelo de enseñanza activo para el alumno, cooperativo y argumentativo, en contraposición con una enseñanza centrada en la organización del discurso del docente como motor de logro de aprendizajes significativos ${ }^{5}$.

\section{5. ¿PARA QUÉ UN NUEVO MODELO DE APREN- DIZAJE?}

La ciencia erudita modeliza sobre recortes de la realidad que se consideran teóricamente relevantes. Cada recorte abstrae, simplifica, reestructura y analoga los diferentes elementos, dando lugar a un sistema en particular. Este sistema, a su vez, es sólo uno de los posibles sistemas que esa porción de realidad seleccionada admite.

Los modelos científicos pasan así a ser representaciones de segundo orden, hechas sobre los sistemas, que ya son en sí mismos abstracciones de la realidad. Así, los modelos resultan representaciones abstractas, escasamente figurativas, más cercanas a una posición abiertamente instrumental que al realismo ingenuo del sentido común (Giere, 1999). En esta postura, los modelos son considerados herramientas de representación teórica del mundo, auxiliares para explicarlo, predecirlo y transformarlo (Adúriz-Bravo, 1999). Entendemos que, así como los científicos piensan su disciplina en base a modelos (Duschl, 1997), los didactas de la ciencia requerimos pensar en un modelo de cómo se aprende, para optimizar nuestras propuestas de enseñanza.

En las últimas décadas, con posterioridad a la propuesta piagetiana, han aparecido numerosos modelos de aprendizaje que se encuentran aún en estudio, evolucionando, y sin ser adoptados por extensos grupos de investigadores. Si bien una revisión profunda del tema excede los alcances del presente artículo, podemos mencionar, además del modelo de aprendizaje significativo de Novak (1999): el modelo de cambio conceptual (Posner, Strike, Hewson y Gertzog, 1982; Hewson, 1992) y su revisión, que tiene en cuenta la ecología conceptual del estudiante (Strike y Posner, 1992); el modelo del aprendizaje generativo de Osborne y Wittrock (1985); el denominado modelos mentales, de Johnson-Laird (1983, 1986); el de las teorías implícitas (Pozo y Gómez, 1998); el de De Posada (1996); el modelo alostérico, de Giordan (1996); y el de De Kleer y Brown (Pintó et al., 1996), proveniente de la inteligencia artificial.

La mayoría de los modelos de aprendizaje tienen en común la aceptación de la importancia de las ideas previas de los alumnos y hacen hincapié en la necesidad de que ellos las replanteen y logren una reestructuración cognitiva o un cambio conceptual. Al intentar relacionar estos modelos de aprendizaje con situaciones de enseñanza cabe preguntarse, por ejemplo:

- ¿Es necesario «dinamitar» los fundamentos que sostienen las concepciones alternativas de los estudiantes? (De Posada, 2000).

- ¿Es posible generar un conflicto cognitivo sobre las ideas previas erróneas de los alumnos sin llegar a apabullarlos?

- ¿Es necesario hacer un relevamiento de todas las ideas previas de los estudiantes para tener en cuenta la ecología conceptual que ronda en cada aula?

- ¿Es epistemológicamente aceptable pensar en procesos de constructivismo educativo para nuestros alumnos basándonos en la necesidad de establecer conflictos cognitivos, que son rupturas epistemológicas?

Nos interesa presentar el MACCS, pues es un modelo que plantea que, a través de situaciones didácticas, habría que ayudar a los estudiantes a encontrar los conceptos ya existentes en sus estructuras cognitivas sobre los cuales pudieran, efectivamente, construir nuevos aprendizajes correctos. El aprendizaje sustentable no supone una ruptura con las ideas previas: propone una construcción sobre los conceptos sostén apropiados.

\section{CONCLUSIONES}

El MACCS es un modelo (Adúriz-Bravo y Galagovsky, 1997; Galagovsky y Adúriz-Bravo, 2001), porque es una simplificación de situaciones complejas que intenta interpretar y predecir situaciones didácticas. Constituye, así, un conjunto de hipótesis que se pondrán a prueba en situaciones reales de clase, aplicables a distintos grupos humanos y contenidos conceptuales y, por lo tanto, a distintos contextos y disciplinas.

El MACCS tiene elementos teóricos que, en forma verbal y gráfica, nos dicen que un aprendizaje sustentable surge al vincular una información como nuevo conocimiento relacionado con conceptos sostén correctos, ya existentes en la estructura cognitiva del alumno. A partir de sus precisiones podemos resignificar y clarificar algunos supuestos, tales como:

- Un contenido significativo no debe ser confundido con un aprendizaje significativo. Un contenido significativo es motivante para los alumnos; en cambio, un aprendiza- 


\section{INVESTIGACIÓN DIDÁCTICA}

je significativo, desde la propuesta de Ausubel y Novak, es un aprendizaje de contenidos nuevos que se relacionan con otros anteriormente aprendidos.

- Guardar información aislada en la memoria de largo plazo no es equivalente a haber construido conocimientos sustentables. Los instrumentos de evaluación deberían poner en evidencia si los saberes de un sujeto son aislados o sustentados; de esta forma, podría distinguirse a posteriori si el proceso de aprendizaje fue aislado o sustentable, respectivamente.

- La ejercitación de la memoria es necesaria aún para la consolidación de conocimientos aprendidos en forma sustentable.

- Errores provenientes de aprendizajes significativos son difíciles de superar. Durante el aprendizaje sustentable deben explicitarse y discutirse los posibles conceptos nexo idiosincráticos hasta encontrar los conceptos sostén apropiados para evitar que se consoliden errores significativos. Errores provenientes de aprendizajes significativos erróneos se constituyen en ideas erróneas persistentes y resistentes al cambio conceptual.

- Los factores afectivos y comunicacionales son el basamento predisponente -positiva o negativamente- para el logro de aprendizajes sustentables, descontando una capacidad cognitiva normal de los sujetos aprendices.

El MACCS, como modelo, nos plantea nuevas cuestiones epistemológicas a resolver teóricamente. Estos planteos son:

- ¿Es lo mismo indagar ideas previas que ayudar a los alumnos a encontrar conceptos sostén?
- ¿Pueden las ideas previas ser sostén de conflictos cognitivos para promover el cambio conceptual?

- ¿Puede lograrse un conflicto cognitivo en los alumnos sin exponerlos públicamente a que expliciten sus ideas erróneas?

- ¿Puede lograrse un cambio conceptual sin pasar por un conflicto cognitivo angustiante para los estudiantes?

Las respuestas a estas preguntas tienen importantes derivaciones comunicacionales y didácticas que serán ampliamente analizadas en la segunda parte del presente trabajo.

\section{NOTAS}

${ }^{1}$ Existen algunas investigaciones que muestran que, en la práctica, muchos alumnos no llegan a comprender conceptualmente un tema aunque éste les sea de interés, tal como el caso de la enseñanza del efecto invernadero y del agujero de ozono (Dove, 1996).

${ }^{2}$ Esta consideración es paralela a la propuesta de Novak, que considera la existencia de un continuo entre aprendizaje significativo y aprendizaje memorístico.

${ }^{3}$ Los contenidos de este nivel preconsciente son accesibles a la conciencia mediante esfuerzos de atención y búsqueda en la MLP.

${ }^{4}$ Los expertos en la disciplina sobre la que versa la información son los que pueden evaluar la corrección de los conceptos a ser utilizados como nexos.

${ }^{5}$ Un aprendizaje receptivo significativo (Novak, 1999) y correcto puede considerarse un caso especial del MACCS, en el cual el sujeto realiza un aprendizaje sustentable en forma autónoma, con el sólo requerimiento de tener acceso a la información que quiere aprender. 


\section{REFERENCIAS BIBLIOGRÁFICAS}

ASUBEL, D. (1968). Educational psychology: A cognitive view. Nueva York: Holt, Rinehart y Winst.

ADÚRIZ-BRAVO, A. (1999). «Elementos de teoría y de campo para la construcción de un análisis epistemológico de la didáctica de las ciencias». Tesis de maestría. Departament de didàctica de las ciències i la matemàtica. Universitat Autònoma de Barcelona.

ADÚRIZ-BRAVO, A. y GALAGOVSKY, L.R. (1997). Modelos científicos y modelos didácticos en la enseñanza de las ciencias naturales. Actas de la X Reunión de Educación en Física. Argentina: Mar del Plata.

CAAMAÑO, A. (1995). La educación CTS: una necesidad en el diseño del nuevo currículo de ciencias. Alambique. Barcelona: Graó.

DE POSADA, J.M. (1996). Hacia una teoría sobre las ideas científicas de los alumnos. Influencia del contexto. Enseñanza de las Ciencias, 14(2), pp. 303-314.

DE POSADA, J.M. (2000). El estudio didáctico de las ideas previas, en Perales Palacios y Cañal de León. Didáctica de las Ciencias Experimentales. Teoría y práctica de la Enseñanza de las Ciencias. Alcoy: Marfil.

DOVE, J. (1996). Students teacher understanding of the green house efect, ozone layer depletion and acid rain. Environmental Education Research, 2(1), pp. 89-100.

DUSCHL, R.A. (1997). Renovar la enseñanza de las ciencias. Importancia de las teorías y su desarrollo. Madrid: Narcea.

FODOR, J.A. (1998). Conceptos. Donde la ciencia cognitiva se equivocó. Madrid: Gedisa.

GALAGOVSKY, L.R. (1993). Redes conceptuales: su base teórica e implicaciones para el proceso de enseñanza-aprendizaje de las ciencias. Enseñanza de las Ciencias, 11(3), pp. 307-311.

GALAGOVSKY, L. y CILIBERTI, L. (1994). Redes conceptuales: su aplicación como instrumento didáctico en temas de física. Enseñanza de las Ciencias, 12(3), pp. 338-349.

GALAGOVSKY, L.R. (1996). Redes conceptuales: memoria, comunicación y aprendizaje (1a. ed). Buenos Aires: Lugar.

GALAGOVSKY, L.R. y ADÚRIZ-BRAVO, A. (2001). Modelos y analogías en la enseñanza de las ciencias naturales. El concepto de modelo didáctico analógico. Enseñanza de las Ciencias, 19(2), pp. 231-242.

GALAGOVSKY, L.R. y MUÑOZ, J.C. (2002). La distancia entre aprender palabras y aprehender conceptos. El entramado de palabras-concepto (EPC) como un nuevo instrumento para la investigación. Enseñanza de las Ciencias, 20(1), pp. 29-45.

GALAGOVSKY, L.R., RODRÍGUEZ, M.A., SANMARTÍ, N. y MORALES, L.F. (2003). Representaciones mentales, lenguajes y codigos en la enseñanza de ciencias naturales. Un ejemplo para el aprendizaje del concepto de reaccion química a partir del concepto de mezcla. Enseñanza de las Ciencias, 21(1), pp. 107-121.
GARDNER, H. (1983). Frames of Mind: The theory of multiples intelligences. Nueva York: Basic Books. Trad. cast. (1995). Inteligencias múltiples. Barcelona: Paidós.

GIERE, R. (1999). Del realismo constructivo al realismo perspectivo. Enseñanza de las Ciencias, núm. extra, pp. 9-13.

GIL, D. (1993). Contribución de la historia y de la filosofía de las ciencias al desarrollo de un modelo de enseñanza-aprendizaje. Enseñanza de las Ciencias, 11(2), pp. 197-212.

GIL PÉREZ, D., CARRASCOSA ALIS, J., DUMAS-CARRÉ, A., FURIÓ MAS, C., GALLEGO, R., GENE DUCH, A., GONZÁLEZ, E., GUISASOLA, J., MARTÍNEZ TORREGROSA, J., PESSOA DE CARVALHO, A.M., SALINAS, J., TRICARICO, H. y VALDÉS, P. (1999). ¿Puede hablarse de consenso constructivista en la educación científica? Enseñanza de las Ciencias, 17(3), pp. 503-512.

GIORDAN, A (1996) ¿Cómo ir más allá de los modelos constructuvistas? La utilización didáctica de las concepciones de los estudiantes. Investigación en la Escuela, 28, pp. 7-22.

HEWSON, P.W. (1992). Conceptual change in science teaching and teacher education. Jornadas sobre «Investigación y Desarrollo del Currículum en la Enseñanza de las Ciencias». Centro Nacional para la Investigación Educativa, la Documentación y la Evaluación. Madrid.

HEWSON, P.W. y HEWSON, M.G.A. (1984). The role of conceptual conflict in conceptual change and design of science instruction. Instructional Science, 13, pp. 1-13. Netherlands: Elsevier.

JIMÉNEZ ALEIXANDER, M.P. (1996). Dubidar para aprender. Vigo: Biblioteca Didáctica.

JOHNSON-LAIRD, P.N. (1983). Mental Models: Towards a Cognitive Science of Language, Inference and Consciuosness. Cambridge, MA: Harvard University Press.

JOHNSON-LAIRD, P.N. (1986). Conditionals and Mental Models, en Traugott E.C., ter Meulen, A., Reilly J.S. y Ferguson, C.A. On Conditionals Cambridge, MA: Oxford University Press.

JOHNSTONE, A.H. (1991). Why is science difficult to learn? Things are seldom what they seem. J. Computer Assisted Learning, 7, pp. 75-83.

JOHNSTONE, A.H. (1999). The nature of chemistry. Education in Chemistry, pp. 45-47.

JORBA, J., GÓMEZ, I. y PRAT, A. (2000). Hablar y escribir para aprender. Uso de la lengua en situaciones de enseñanza-aprendizaje desde las áreas curriculares. Madrid: Síntesis - ICE de la UAB.

LAWSON, A.E. (1982). The reality of general cognitive operations. Science Education, 66, pp. 229-241.

LEMKE, J. (1997). Aprender a hablar ciencia. Barcelona: Paidós.

MUÑOZ, J.C. (2000). «Entramado de palabras-concepto: un instrumento para el análisis de contenido». Tesis de licen- 
ciatura en enseñanza de las ciencias. Universidad de General San Martín. Buenos Aires.

MAYER, R.E. (1985). El futuro de la psicología cognitiva. Madrid: Alianza.

MORENO, M. (1988). Imaginación y ciencia, en Moreno, M. (ed.). Ciencia, aprendizaje y comunicación. Barcelona: Laia.

NOVAK, J.D. (1984). Teoría y práctica de la educación. Madrid: Alianza.

NOVAK, J.D. (1999). Conocimiento y aprendizaje. Los mapas conceptuales como herramientas facilitadoras para escuelas y empresas. Madrid: Alianza.

OGBORN, J., KRESS, G., MARTINS, I. y McGILLICUDDY, K. (1998). Formas de explicar. La enseñanza de las ciencias en secundaria. España: Aula XXI - Santillana.

OSBORNE, R.J. y WITTROCK, M.C. (1985). The generative learning model and its implications for science education. Studies in Science Education, 12, pp. 59-87.

PERALES PALACIOS, F.R y CAÑAL DE LEÓN, P. (2000). Didáctica de las ciencias experimentales. Teoría y práctica de la enseñanza de las ciencias. Alcoy: Marfil.

PINTÓ, R., ALIBERAS, J. y GÓMEZ, R. (1996). Tres enfoques de la investigación sobre concepciones alternativas. Enseñanza de las Ciencias, 14(2), pp. 221-232.

PORLÁN, R. (1998). Pasado, presente y futuro de la didáctica de las ciencias. Enseñanza de las Ciencias, 16(1), pp. 175185.

POSNER, G.J, STRIKE, K.A., HEWSON P.W.A. y GERTZOG, W.A.(1982).Accomodation of a scientific conception:Towards a theory of conceptual change. Science Education, 66(2), pp. 211-227.

POZO, J.I. (1987). La historia se repite: las concepciones espontáneas sobre el movimiento y la gravedad. Infancia y aprendizaje, 38, pp. 69-87.

POZO, J.I. (1997). Teorías cognitivas del aprendizaje. Madrid: Morata.

POZO, J.I. (2001). Humana mente. El mundo, la conciencia y la carne. Madrid: Morata.

POZO, J.I. y GÓMEZ, M.A. (1998). Aprender y enseñar ciencia. Del conocimiento cotidiano al conocimiento científico. Madrid: Morata.

RIVIERE, A. (1986). Razonamiento y representación. Madrid: Siglo XXI.

RODRÍGUEZ, M.A. (2001). «Un estudio sobre la vinculación entre el lenguaje utilizado en clase y las representaciones construidas por los estudiantes durante el aprendizaje del tema reacciones químicas». Tesis de licenciatura en enseñanza de las ciencias. Universidad de General San Martín. Buenos Aires.

SOLBES, J. y VILCHES, A. (1989). Interacciones ciencia - tecnología - sociedad. Un instrumento de cambio actitudinal. Enseñanza de las Ciencias, 7(1), pp. 14-20.

STRIKE, G. y POSNER, G. (1992). A revisionist theory of conceptual change, en Duschl, R.A. y Hamilton, R.J. (eds.). Phylosophy of science, cognitive psychology and educational theory and practice, pp. 147-176. Albany, Nueva York: State University of New York Press.

WHITE, R.T. (1988). Learning Science. Oxford, UK: Basil Blackwell Ltd. 